\title{
Very high energy gamma-rays from flat spectrum radio quasars
}

\author{
Elina Lindfors, on behalf of the MAGIC Collaboration
}

Tuorla Observatory, University of Turku, Finland

email: elilin@utu.fi

\begin{abstract}
The detection of Flat Spectrum Radio Quasars (FSRQs) in the Very High Energy (VHE, E $>100 \mathrm{GeV}$ ) range is challenging, mainly because of their steep soft spectra and distance. Nevertheless four FSRQs are now known to be VHE emitters. The detection of the VHE $\gamma$-rays has challenged the emission models of these sources. The sources are also found to exhibit very different behavior. I will give an overview of what is known about the VHE emission of these sources and about the multiwavelength signatures that are connected to the VHE gamma-ray emission.
\end{abstract}

Keywords. gamma rays: observations, quasars: individual (3C 279, PKS 1510-089, PKS 1222+216)

\section{Introduction}

Blazars, that are the most numerous sources in the extragalactic VHE $\gamma$-ray sky, are a common name for BL Lac objects and flat spectrum radio quasars (FSRQs). Blazars host relativistic jets that are pointing close to our line of sight. Blazars show variable emission in all wavebands from radio to $\gamma$-rays, but in FSRQs the variability is typically larger in amplitude (Abdo et al. 2010a). VHE $\gamma$-ray FSRQs are more luminous in MeV-GeV band than the VHE $\gamma$-ray emitting BL Lac objects, and in general also more distant (Abdo et al. 2010b).

The spectral energy distributions (SED) of both subclasses of blazars show two peaks: first peak is generally attributed to synchrotron emission and the second to inverse Compton scattering. The seed photons for the inverse Compton scattering are provided by the synchrotron emission (e.g. Maraschi et al. 1992) or can originate from the dense radiation field generated by the direct and reprocessed accretion disk emission (Dermer \& Schlickeiser 1994, Sikora et al. 1994) or molecular torus (Sikora et al. 2008). Also hadronic mechanisms for producing the second peak have been proposed (e.g. Böttcher et al. 2009).

In the case of FSRQs, the first SED peak is typically in the infrared regime while for BL Lacs it is between optical and hard X-rays. The optical spectra of FSRQs show broad emission lines, implying an existence of fastly moving gas clouds close (0.1 to 1 parsec) to the central engine, while BL Lacs show very weak or no emission lines in their spectra. Because of these properties, FSRQs were considered not to be good candidates to emit VHE $\gamma$-rays: the low synchrotron peak frequency implies efficient cooling (e.g. Ghisellini et al. 1998), which makes it difficult to produce VHE $\gamma$-ray emission. Moreover, the broad line region clouds absorb the $\gamma$-ray emission via pair production. Additionally, the large redshift also means stronger absorption of VHE $\gamma$-rays by the extragalactic background light on their way to earth. However, four FSRQs have been detected by the Imaging Air Cherenkov Telescopes (IACTs): 3C 279 (Albert et al. 2008), PKS 1510-089 (Abramowski et al. 2013, Aleksic et al. 2014b), PKS 1222+216 (Aleksic et al. 2011, Holder 2014) and S3 0218+357 (Mirzoyan 2014). Moreover, in the Fermi-LAT > $10 \mathrm{GeV}$ source catalog 70 FSRQs are listed (Ackermann et al. 2013). 


\section{Very high energy gamma-rays}

Despite the large number of hours spent on FSRQs by the current generation of telescopes, there are only a handful of detections of VHE $\gamma$-rays from FSRQs from the IACTs. All the detections show soft spectra, but the observed fluxes and variability timescales differ dramatically.

3C 279 has been detected twice in VHE $\gamma$ rays, on February 23, 2006 and January 16, 2007 (Albert et al. 2008). Both detections come from the MAGIC Telescopes. All the other observations of 3C 279 by MAGIC and VERITAS (Errando et al. 2011) have resulted in upper limits. The most recent upper limits are below the detected flux confirming that the source was detected in a high state (Aleksic et al. 2014a). The flux and spectra of the two observations are comparable within the error bars, the 2006 measured integral flux being $\mathrm{F}(>150 \mathrm{GeV})=5.15 \pm 0.82_{\text {stat }} \pm 1.5_{\text {syst }} \times 10^{-10}$ photons $\mathrm{cm}^{-2} \mathrm{~s}^{-1}$ and spectral index $\alpha=4.1 \pm 0.7_{\text {stat }} \pm 0.2_{\text {syst }}$. Also the variability appears similar: the significant detection is preceded by a hint of signal on 2-3 $\sigma$ level from the previous night. For the 2006 observation there was no observation from the following night, but in 2007, there is no signal in the data of following night.

PKS 1222+216 was detected in VHE $\gamma$-rays on June 17, 2010 by the MAGIC telescope. The source had been monitored by the MAGIC telescope since the beginning of May 2010, the data from May 8, 2010 showing a hint of signal at $3-4 \sigma$ level. The MAGIC observations on June 17, 2010 had a duration of only 30 minutes, but even within this short observation the flux was significantly variable with the flux doubling time of order of $\sim 10$ minutes (Aleksic et al. 2011). The observed spectral slope is soft with $\Gamma=3.75 \pm 0.29$. Recently VERITAS reported a detection of a source with signal coming from several nights rather than from a single night strong flare (Holder 2014).

PKS 1510-089 has also been detected twice in VHE $\gamma$ rays in two extended periods from March and April 2009 by H.E.S.S. (Abramowski et al. 2013) and February-April 2012 by MAGIC (Aleksic et al. 2014b). The flux and spectra of the two observations agree within the error bars. The integral flux of the 2012 measurement is $\mathrm{F}(>200 \mathrm{GeV})=$ $(3.6 \pm 0.9) \times 10^{-11} \mathrm{~cm}^{-2} \mathrm{~s}^{-1}$. The best-fit power law to the VHE data has a photon index of $\Gamma=3.8 \pm 0.4_{\text {stat }} \pm 0.3_{\text {sys }}$. There is no published upper limits of the source below these detections and therefore it cannot be concluded if the detected flux presents high or quiescent state of the source at VHE $\gamma$-rays. Neither of the data sets show statistically significant variability in daily, weekly or monthly scales. It should be noted, however, that in the Fermi Hard source catalog this source is listed as variable above $>10 \mathrm{GeV}$ (Ackermann et al. 2013).

S3 0218+357 is the fourth FSRQ discovered in VHE $\gamma$-rays. It is a gravitationally lensed blazar located at the redshift of $0.944 \pm 0.002$ and was discovered by MAGIC in July 2014 (Mirzoyan 2014). The data analysis is ongoing.

\section{Multiwavelength behavior during VHE gamma-ray detections}

All FSRQs were in a high state in the lower energy regimes every time they were detected at VHE $\gamma$-rays. However, the activity has not followed the same patterns during the four VHE detections. Moreover, some of the observations in high optical and $\gamma$-ray states have resulted in flux upper limits.

3C279: Both VHE $\gamma$-ray detections took place before the launch of AGILE and FermiLAT, so there are no simultaneous HE $\gamma$-ray data available. The radio, optical, X-ray and VHE $\gamma$-ray lightcurves of these two epochs are shown in Fig. 1. Compared to quiescent state of the source, they show a factor of two brightening in X-ray band at the time of 2006 detection and factor of nine brightening in optical at the time of 2007 detection. 

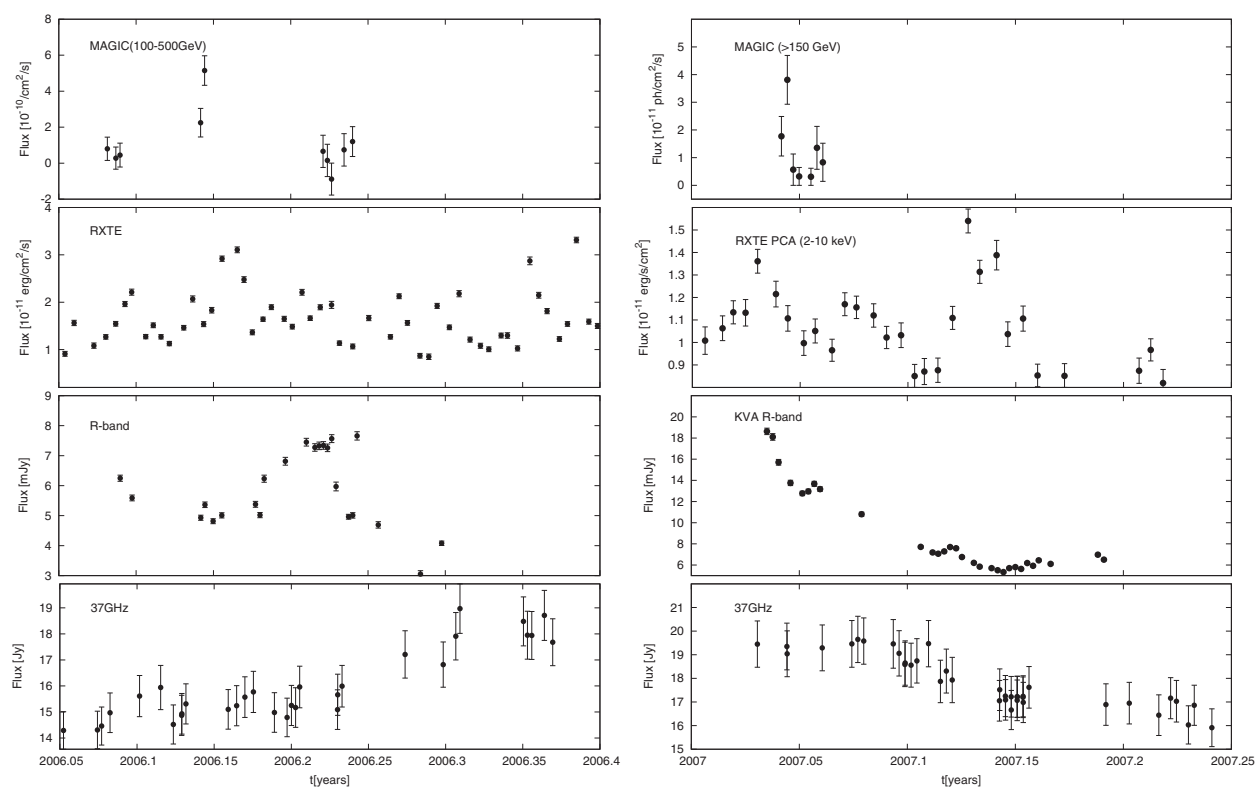

Figure 1. The multiwavelengths lightcurve of 3C 279 from January to May 2006 (left) and from January to March 2007 (right). Left: In February 2006, at the time of the detection of the VHE gamma-rays, the X-ray flux and optical fluxes are variable and the lightcurve showed small peaks (factor of two for X-rays, factor of $50 \%$ in optical, compared to typical quiescent state of the source) around the time of the VHE $\gamma$-ray detection. The main flares in X-ray (factor of three) and optical (factor of two) take place after the VHE detection, with delays of $\sim 18$ days and 35 days respectively. The $37 \mathrm{GHz}$ lightcurve shows no variability during the time of the VHE $\gamma$-ray detection, the flux increase starts when the optical outburst is already decreasing. There was a new VLBA component emerging from the $43 \mathrm{GHz}$ VLBA core at 2006.45 (Chatterjee et al. 2008). There is a delay of 4 months from VHE to radio event, so these events are not necessary linked to each other. Right: In January 2007 the observed MWL behavior is very different compared to 2006. The X-ray flux in general is low, with maximum flux increase of $50 \%$. There was a major radio and optical outburst, with peak flux $>2$ times higher than the 2006 outburst. At the time of the VHE $\gamma$-ray detection the flare was already decreasing. The radio-optical outburst was accompanied by the ejection of the new component from the $43 \mathrm{GHz}$ VLBA core and simultaneous rotation of the electric vector position angle (EVPA) of the optical polarization and the polarization of the VLBA core (Larionov et al. 2008).

There are also more recent observations of 3C 279. In the spring of 2011 3C 279 was showing increasing optical flux $(\mathrm{F}>3 \mathrm{mJy})$, as well as increased $\gamma$-ray flux as observed by Fermi-LAT $\left(\mathrm{F}>10^{-6} \mathrm{~cm}^{-2} \mathrm{~s}^{-1}\right)$, which triggered MAGIC observations of the source. The multiwavelength light curves from radio to $\gamma$-rays (Fig. 2, left) show complex behavior. There is significant variability in all light curves with several maxima in the lightcurves. The optical flux start to increase around the time of second maxima in $\gamma$ - and X-ray light curves. It is accompanied by an increase in the optical polarization and a rotation of the polarization angle. While the MAGIC observations missed the largest $\gamma$-ray peak, there was still a small $\gamma$-ray flare (factor of two) in this epoch, and the optical flux reached its maxima (factor of two) during the MAGIC observing period. In optical, this event is the third brightest since the beginning of the Tuorla monitoring program ( $>10$ years of data; http://users.utu.fi/kani/1m). There are similarities to the behavior in 2007 (in particular the rotation of the EVPA), but the maxima of the optical lightcurve is factor of 3 smaller and there was no new component ejected from $43 \mathrm{GHz}$ VLBA core. As MAGIC observations did not yield in a significant signal, it seems that in the case of $3 \mathrm{C} 279$, 
lower energy flaring events that provoke the emission of VHE $\gamma$-rays are rather rare (see Aleksic et al. 2014a for further discussion).

PKS 1510-089: The first detection of the VHE $\gamma$-rays took place in March 2009 when the source was active in the HE $\gamma$-ray band. In HE $\gamma$-rays the flaring event consisted of several flares. In X-rays the flaring was moderate and not correlated with the $\gamma$-ray flaring, but in optical the last $\gamma$-ray flare was accompanied by a large optical outburst (factor of nine) and a large radio outburst (Marscher et al. 2010). During the $\gamma$-ray flares the optical polarization angle was rotating by $>720^{\circ}$ and during the major optical flare, the optical polarization increased to $>30 \%$. In the $43 \mathrm{GHz}$ VLBA maps a superluminal knot emerged from the VLBA core with a zero-separation epoch essentially simultaneous with this bright optical flare. However, the main part of the H.E.S.S. observations took place before the rotation of the optical polarization angle started. There are no VHE gamma-ray observations from the period when the knot passed the VLBA core. The connection between the gamma-ray flaring, rotation of EVPA, and emerging of new VLBA components suggests common emission region $>10 \mathrm{pc}$ from the central engine (Marscher et al. 2010).

Fig. 2 (right) shows multiwavelength light curve from spring 2012, the epoch of MAGIC observations. It has several similarities to the event in gamma-rays in 2009: ejection of the knot from the VLBA core, accompanied activity in the millimetre wavelengths and the rotation of the optical polarization angle. However, there are also some differences: there is no preceding gamma-ray flare (and it should be noted that the H.E.S.S. observations in 2009 took place during this flare), but the activity in radio and $\gamma$-rays starts rather simultaneously. Also the observed rotation of the optical polarization angle is shorter in duration $(\sim 30$ days) and the rotation is only $\sim 350$ degrees instead of $>720$ degrees seen in 2009. However, with the significant similarities, the far from black hole emission region is suggested also for this flaring event (Aleksic et al. 2014b).

PKS 1222+216: The MAGIC VHE $\gamma$-ray observations of PKS $1222+216$ were triggered by the bright flaring at the HE $\gamma$-ray band, reported by Fermi-LAT (Donato et al. 2010). The gamma-ray activity was accompanied by a bright optical flare and the X-ray flux was relatively low and the variability was only moderate (Ackermann et al. 2014). There was a VLBA component ejected from the $43 \mathrm{GHz}$ VLBA core on $\sim$ May 14, 2010 (Marscher et al. 2012). It appears that the MWL behavior was somewhat similar to the PKS 1510-089 behavior in spring 2012 with several HE $\gamma$-ray flares, low state with little variability in X-rays and bright optical flaring. In the spring 2014, the VHE $\gamma$-ray emission was again accompanied with the ejection of new VLBA component (Jorstad et al. 2015).

\section{Spectral energy distributions}

The SEDs of FSRQs are typically modeled with a small emission region close to the central engine, in regions where the dense radiation field from the broad line region clouds is thought to provide the ideal environment for an efficient inverse Compton emission (e.g. Hartman et al. 2001). The VHE $\gamma$-ray detections challenge this view because the combined HE to VHE $\gamma$-ray SEDs show no signature of a break Aleksic et al. 2011, Aleksic et al. $2014 \mathrm{~b}$ as would be expected if the emission took place inside the broadline region (e.g. Donea \& Protheroe 2003). This makes the molecular torus a more appealing source of seed photons in the periods of VHE $\gamma$-ray emission (Tavecchio et al. 2011, Barnacka et al. 2014). The apparent connection between some of the flares with the ejection of new components from the $43 \mathrm{GHz}$ VLBA core might place the emission region even further down the jet, where the external seed photons might be provided by the slow sheath of the jet (Marscher et al. 2010). We investigated these two possibilities 

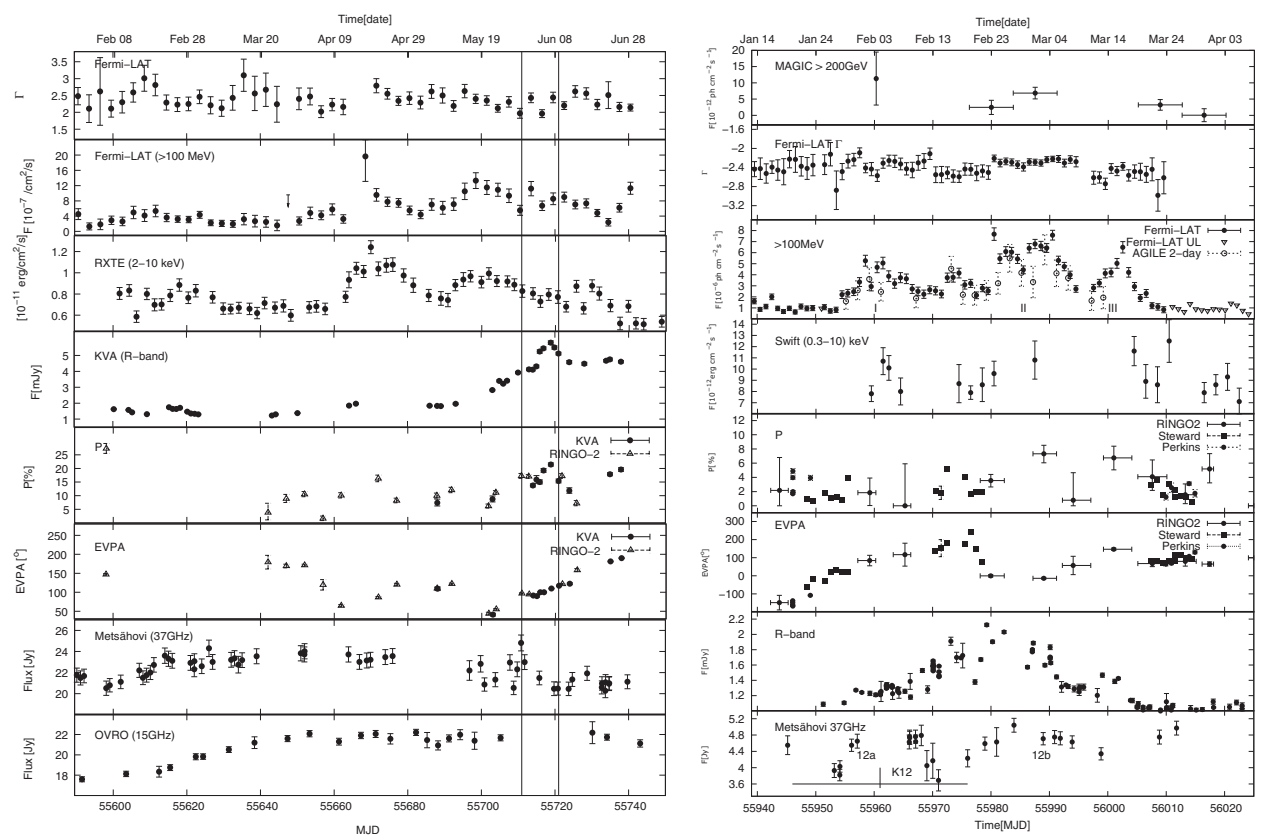

Figure 2. The MWL light curve of 3C 279 in 2011 (left) and PKS 1510-089 in 2012 (right) from radio to $\gamma$-rays. In these epochs the sources showed similar behavior in optical (flare and rotation of the EVPA) and Fermi-LAT band (multiple flares), but 3C 279 observations resulted in no detection with MAGIC (the vertical lines in left figure indicate the period of the MAGIC observations), while for PKS 1510-089 VHE gamma-ray emission was detected. In the right figure the symbol marked K12a indicates the time of the ejection of a new component from the $43 \mathrm{GHz}$ VLBA core of PKS 1510-089, while for 3C 279 no new component was resolved. Therefore we suggested for $3 \mathrm{C} 279$, that the gamma-ray flaring region was close to central engine and VHE $\gamma$-ray emission was absorbed, while for PKS 1510-089, the gamma-ray flaring emission is close to VLBA core far from the central engine and therefore VHE $\gamma$ rays were observed. The figures are adapted from (Aleksic et al. 2014a) and (Aleksic et al. 2014b).

in the case of PKS 1510-089 and concluded that both result in an acceptable fit to the average SED (Aleksic et al. 2014b) and we cannot resolve them.

Even if the "far from central engine" emission scenarios for the VHE $\gamma$-ray emission have the benefit of avoiding the strong absorption from the broad line region, there is also a severe draw back. The HE and VHE $\gamma$-ray emissions show very fast variability with variability time scales as short as 10 minutes. This means that the emission region must be very compact, significantly smaller than the cross-section of the parsec scale jets (Tavecchio et al. 2011). There have been several suggestions how to solve this dilemma, among them, e.g. the jet-in-jet model (Giannios et al. (2009)).

\section{Summary and Conclusions}

Our current knowledge of the VHE $\gamma$-ray emission from FSRQs relies on observations of three FSRQs: 3C 279, PKS 1510-089 and PKS 1222+216. While these observations are certainly not comprehensive, there are several lessons that we have learned:

- FSRQs do emit VHE $\gamma$-rays. This fact alone (even without the complex MWL signatures) is challenging to model with the traditional one-zone inverse Compton models, where the emission region is close to the black hole.

- The fast variability (10 $\mathrm{min})$ in PKS $1222+216$ requires a compact emission region.

- VHE $\gamma$-ray flares from FSRQs have been detected only during "high" states in the 
lower energy regimes, with a high state defined here as factor of two increase in flux in at least one of the monitored wavebands (optical, X-ray HE $\gamma$-rays).

- At least for 3C 279 and PKS 1222+216 the VHE $\gamma$-ray flux outside these high states seems to be below the sensitivity of the current instruments.

- In several cases the VHE $\gamma$-ray emission is observed in a period coincident with ejection of new component from the $43 \mathrm{GHz}$ VLBA core and rotation of the EVPA of optical polarization. It indicates that VHE $\gamma$-rays are emitted far from the central engine close to the $43 \mathrm{GHz}$ VLBA core.

However, the VHE $\gamma$-ray observations of FSRQs are very few and the improved sensitivity of CTA is necessary in order to increase the number of VHE $\gamma$-ray detections. These would be used to understand the overall phenomena better: even with only three sources we see great variety in the variability behavior, which should, however, represent only the tip of the iceberg of the whole VHE $\gamma$-ray emitting population. Moreover, combined with lower frequency monitoring, the CTA observations would allow tackling specific science questions in particular the location of the VHE $\gamma$-ray emitting region.

This paper has made use of published multiwavelength data from MAGIC (http://magic.mppmu.mpg.de), Fermi-LAT, Swift and RXTE X-ray satellites, KVA, ATOM and Perkins optical telescopes, Metsähovi Radio Observatory and Owens Valley Radio Observatory.

\section{References}

Abdo, A. A., Ackermann, M., Ajello, M., et al. 2010a, ApJ, 722, 520

Abdo, A. A., Ackermann, M., Ajello, M., et al. 2010b, ApJ, 715, 429

Abramowski, A., et al. 2013, A\& A, 554, 107

Ackermann, M. et al. (Fermi-LAT Collaboration) 2013, ApJS, 209,34

Ackermann, M. et al. (Fermi-LAT Collaboration) 2014, ApJ, 786, 157

Albert, J., et al. (MAGIC Collaboration) 2008, Science, 320, 1752

Aleksic, J., et al. (MAGIC Collaboration) 2011, ApJ, 730, L8

Aleksic, J., et al. (MAGIC Collaboration) 2012, APh, 35, 435

Aleksic, J., et al. (MAGIC Collaboration) 2014a, A\&A, 567, 41

Aleksic, J., et al. (MAGIC Collaboration) 2014b, A\&A, 569, 46

Barnacka, A., Moderski, R., Behera, B., Brun, P. \& Wagner, S. 2014 A $\& A$, 567, 113

Böttcher, M., Reimer, A., \& Marscher, A. 2009, ApJ, 703, 1168

Chatterjee, R., Jorstad, S. G., Marscher, A. P. et al. 2008, ApJ, 689, 79

Dermer, C. \& Schlickeiser, R., 1994, ApJS, 90, 945

Donato, D. et al. (Fermi-LAT collaboration) 2010, Astronomer's Telegram \#2584

Donea, A.-C. \& Protheroe, R. J. 2003, APh, 18, 377

Errando M. et al. (VERITAS collaboration) 2011, Proc. 32nd ICRC, Beijing, China

Ghisellini, G. et al. 1998, MNRAS, 301, 451

Giannios, D., Uzdensky, D. A., \& Begelman, M. C. 2009, MNRAS, 395, L29

Hartman, R. C., Böttcher, M., Aldering, G. et al. 2001, ApJ, 553, 683

Holder, J. (on behalf of the VERITAS collaboration) 2014, Astronomer's Telegram \#5981

Jorstad, S. et al. 2015, these proceedings

Larionov, V. et al. 2008, A\&BA, 492, 389

Maraschi L. et al. 1992, ApJ, 397, 5

Marscher, A., Jorstad, S., Larionov, V. et al. 2010, ApJL, 710, L126

Marscher, A. et al. 2012, Proc. Fermi \& Jansky, eConf C1111101, arXiv:1204.6707

Mirzoyan, R. (on behalf of the MAGIC collaboration) 2014, Astronomer's Telegram \#5887

Sikora, M., Begelman, M. C., \& Rees, M. J. 1994, ApJ, 421, 153

Sikora, M., Moderski, R., \& Madejski, G. M., 2008, ApJ, 675, 71

Tavecchio, F. et al. 2011, A\&A, 534, 86 